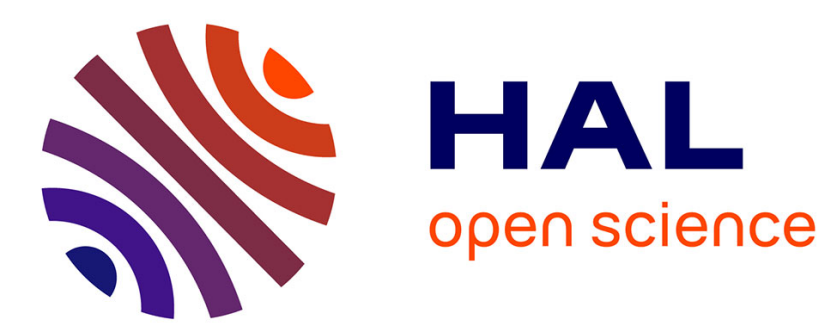

\title{
Viscoelasticity Breaks the Symmetry of Impacting Jets
}

Henri Lhuissier, Baptiste Néel, Laurent Limat

\section{To cite this version:}

Henri Lhuissier, Baptiste Néel, Laurent Limat. Viscoelasticity Breaks the Symmetry of Impacting Jets. Physical Review Letters, 2014, 113, 10.1103/PhysRevLett.113.194502 . hal-01441563

\section{HAL Id: hal-01441563 https://hal.science/hal-01441563}

Submitted on 25 Jan 2017

HAL is a multi-disciplinary open access archive for the deposit and dissemination of scientific research documents, whether they are published or not. The documents may come from teaching and research institutions in France or abroad, or from public or private research centers.
L'archive ouverte pluridisciplinaire HAL, est destinée au dépôt et à la diffusion de documents scientifiques de niveau recherche, publiés ou non, émanant des établissements d'enseignement et de recherche français ou étrangers, des laboratoires publics ou privés. 


\title{
Viscoelasticity Breaks the Symmetry of Impacting Jets
}

\author{
H. Lhuissier, B. Néel, and L. Limat \\ Matière et Systèmes Complexes, Université Paris Diderot, CNRS UMR 7057, 10 rue Alice Domon et Léonie Duquet, \\ 75205 Paris, France
}

(Received 6 August 2014; published 7 November 2014)

\begin{abstract}
A jet of a Newtonian liquid impacting on a wall at right angle spreads as a thin liquid sheet which preserves the radial symmetry of the jet. We report that for a viscoelastic jet (solution of polyethylene glycol in water) this symmetry can break; close to the wall, the jet cross section becomes faceted and radial steady liquid films (wings) form, which connect the cross-section vertices to the sheet. The number of wings increases with increasing the viscoelastic relaxation time of the solution, but also with increasing jet velocity and decreasing distance from the jet nozzle to the wall. We propose a mechanism for this surprising destabilization of the jet shape, which develops perpendicularly to the direction expected for a buckling mechanism, and explain these dependencies. We also discuss the large-scale consequences of the jet destabilization on the sheet spreading and fragmentation, which show through the faceting of hydraulic jumps and of suspended (Savart) sheets.
\end{abstract}

PACS numbers: 47.60.Kz, 47.20.Ky, 47.50.-d, 83.50.Lh

Since Savart's thorough study [1], the impact of a jet on a solid surface has motivated many works to understand the pressure and the shear on impact [2-4], the liquid deflection into a wetting or suspended liquid sheet [1], and the circular hydraulic jump [5,6], or receding sheet edge [7,8], that forms downstream. These situations have direct applications for cutting [9] and cleaning with high pressure jets, cooling by jet impingement [10], and atomizing liquids [8,11,12]. However, very little attention has been paid to the important case of viscoelastic liquids $[13,14]$. These liquids are ubiquitous in nature (mucus, DNA, tree sap) and in human activities (adhesives, drag reduction additives, paints, propellants). Contrarily to Newtonian liquids, they develop anisotropic elastic stresses (normal stresses) in a flow, which result in unusual steady phenomena (e.g., rod climbing, die swell, and open siphon $[15,16])$, but also dynamic instabilities and noninertial turbulence $[15,17,18]$. We study here the impact of a viscoelastic jet and report a new phenomenon: the steady symmetry breaking of the jet on impact, and its consequences on the spreading and fragmentation of the liquid sheet downstream.

Our experiment consists of a viscoelastic liquid jet impacting perpendicularly on a smooth glass plate. The jet is formed by extruding the liquid from a large $(20 \mathrm{~L})$ pressurized tank, either through a long tranquilization chamber (stainless steel tube) ended by a cone-shaped stainless steel nozzle, or through a Pasteur pipette (essentially straight glass nozzle). In both case a straight jet with radius $a$ is obtained (no significant contraction nor swell is observed). The mean flow velocity $u$ in the jet is set by adjusting the constant air pressure in the tank with a pressure regulator and continuously monitoring the tank weight $(1 \mathrm{~g}$ precision scale AIP System). The distance $l$ from the nozzle exit to the plate is varied with a calibrated microcontrolled linear stage. We used several liquids (see below), but for the systematic study we focused on a solution of unbranched polymer in distilled water (polyethylene glycol [PEO Sigma-Aldrich] with molecular weight $M=4 \times 10^{6} \mathrm{~g} / \mathrm{mol}$ at a concentration of $2 \mathrm{~g} / \mathrm{L}$ ). The solution of an air pressure driven flow is chosen to limit the mechanical degradation of the viscoelastic solution in a pump prior to its impact. It imposes a cyclic process: the whole liquid is extruded and collected downstream from the impact to be poured back to the tank for a new cycle. In spite of the pumpfree system, the solution slowly evolves from cycle to cycle due to the large shear stress on impact. At each cycle a sample is collected to measure the rheological properties of the solution [19]. The viscosity is essentially independent from the aging of the solution and, in the model below, we will consider an effective shear viscosity $\eta=5 \mathrm{mPas}$ that is relevant for the typical shear rate $u / a \sim 10^{3}-10^{4} \mathrm{~s}^{-1}$ in the experiments. The relaxation time $\lambda$ of the solution is measured from the constant time scale of the thinning dynamics of the filament that is left behind a detaching drop [20]. It is found to regularly decrease (from 17.6 to $7.3 \mathrm{~ms}$ ) with the aging of the solution. The solution has a density $\rho=995 \mathrm{~kg} / \mathrm{m}^{3}$ (measured with a calibrated flask) and a surface tension $\gamma=60 \mathrm{mN} / \mathrm{m}$ (measured by fitting the static shape of a pendant drop).

Depending on the impact conditions (velocity $u$ and plate-to-nozzle distance $l$ ), the viscoelastic properties of the liquid strongly affects the jet deflection and spreading on the plate, as illustrated in Fig. 1. At low $u$ and large $l$, the jet behaves as a Newtonian jet [Fig. 1(b)]. The radial symmetry of the jet is preserved, both at the impact and in the spreading, and the deflection of the liquid is confined to a small region with size comparable to the jet radius. At large $u \gtrsim 1 \mathrm{~m} / \mathrm{s}$ and short $l \lesssim 1 \mathrm{~cm}$, the impact is also steady but its structure changes: The radial symmetry breaks [Fig. 1(a)]. 


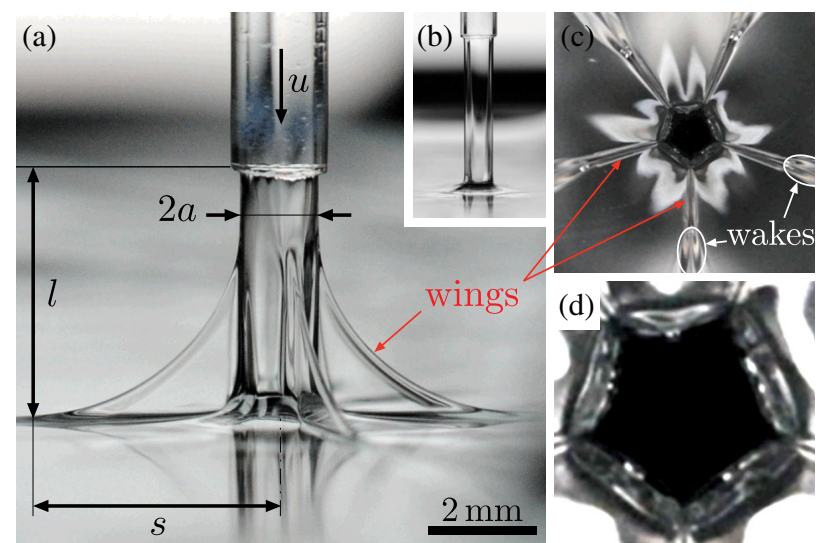

FIG. 1 (color online). Impact of a viscoelastic jet (PEO $4 \times$ $10^{6} \mathrm{~g} / \mathrm{mol}$ at $2 \mathrm{~g} / \mathrm{L}$ in water, $a=0.71 \mathrm{~mm}$, mean velocity $u=8.46 \mathrm{~m} / \mathrm{s}$ ) extruded from a Pasteur pipette onto a glass plate. (a),(c),(d) At small plate-to-nozzle distance $l$, steady triangular radial liquid films (wings) connect the jet to the sheet that spreads on the plate. (a) Side view showing three of the five wings (the jet image at the bottom is a reflection on the sheet). (c),(d) Bottom view through the plate. Close to the plate, the jet has a cusped pentagonal cross section (appearing in black). The five equally spaced wings initiate at the cross-section vertices and generate a wake downstream on the sheet. (b) For larger $l$ no wing forms.

Close to the plate, planar triangular radial liquid films, which we call wings, form around the jet. They attach both to the jet and to the spreading liquid sheet. A bottom view through the transparent impact plate [Figs. 1(c) and 1(d)] reveals important features of the impact structure in the latter case. The wings are equally spaced around the jet (from 3 to 34 wings, the maximum we could observe). Close to the plate, the jet cross section actually has a cusped polygonal shape, with the wings attaching to the polygon vertices. Between two consecutive wings a bright region is often observed. We speculate that it is a manifestation of the birefringence due to anisotropy of the polymers in the flow since no curvature of the interface is resolved at that place. A bright region is also observed immediately downstream of the wings, where a wake forms in the sheet that has a long-range influence on the sheet (see below). For a given viscoelastic liquid, the number of wings $n$ increases with increasing velocity $u$ and decreasing plate-to-nozzle distance $l$, as shown in Fig. 2. Similarly, the size $s$ of the wings [see Fig. 1(a)] increases with increasing $u$ and decreasing $l$, except at short distances when the wings are confined between the nozzle and the plate and their size is set by $l$ (see Fig. 2 and the Supplemental Material A [22]).

This destabilization of the jet (and its dependency on $u$ and $l$ ) is a robust phenomenon. It strongly depends on the viscoelasticity of the liquid but only weakly on the other parameters we could modify. First, the symmetry breaking is only observed for solutions of polymers with high molecular weights (PEO with $M=1$ to $8 \times 10^{6} \mathrm{~g} / \mathrm{mol}$
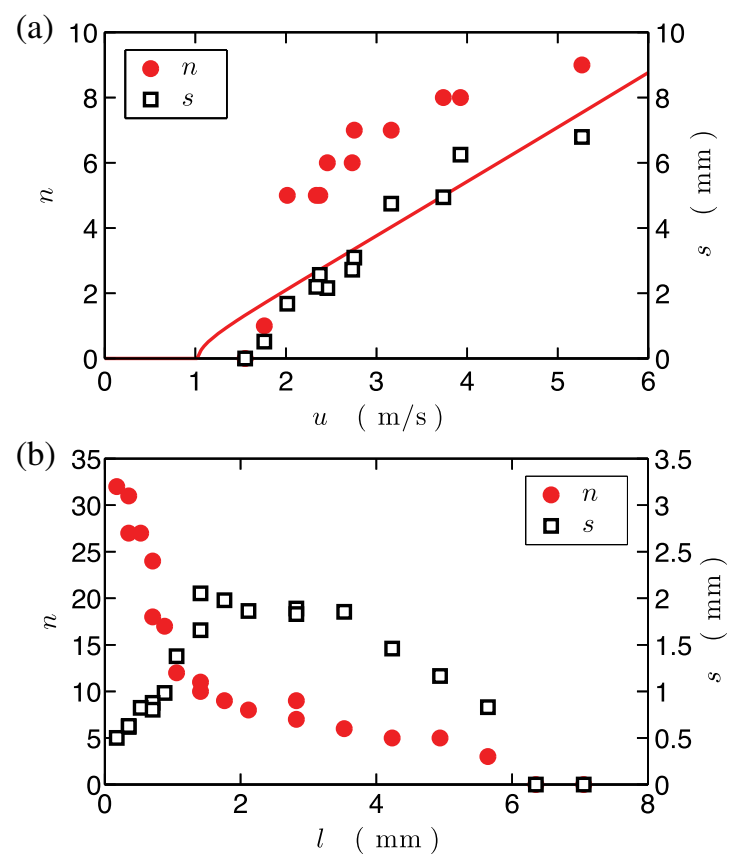

FIG. 2 (color online). Typical evolution of the number $n$ and size $s$ of the wings versus, (a) the mean jet velocity [ $a=1.38 \mathrm{~mm}, l=3.75 \mathrm{~mm}$, and $\lambda=9.6 \mathrm{~ms}$. The red solid line is the prediction for $n$ from Eq. (2)], and (b) the plateto-nozzle distance $l \quad(a=1.38 \mathrm{~mm}, \quad u=3.07 \mathrm{~m} / \mathrm{s}$, and $\lambda=8.2 \mathrm{~ms}$ ).

and polyacrylamide $\left.18 \times 10^{6} \mathrm{~g} / \mathrm{mol}\right)$ at concentrations $(\sim 1 \mathrm{~g} / \mathrm{L}) \quad$ where significant relaxations times $(\lambda \sim 10-100 \mathrm{~ms})$ are measured. As a solution ages its elastic properties decay ( $\lambda$ decreases) and we observed that the velocities and distances required to form wings respectively increase and decrease. By contrast, no symmetry breaking could be observed for any Newtonian liquid we tried (water-glycerol solutions with viscosity up to $80 \mathrm{mPa} s$ and velocity $u>10 \mathrm{~m} / \mathrm{s}$ ), nor for saturated water solutions of commercial surfactants. This suggests that the instability is neither a viscous buckling nor a Marangoni effect that would be exacerbated by elastic stresses, but rather relies on a purely viscoelastic mechanism. Second, for a given viscoelastic solution, the destabilization is observed in many different impact situations with similar dependencies on $u$ and $l$ (see the Supplemental Material B [22]): facing circular jets (i.e., coaxial jets with same size and opposite velocities), a circular jet impacting obliquely on a plate, and the perpendicular impact of an elongated rectangular jet [i.e., a liquid sheet with a large width-to-thickness ratio; see Figs. 3(a) and 3(b)]. The first case shows that the no-slip condition on the plate is not mandatory to the destabilization, and the third case indicates that the radial radial symmetry is also not necessary.

In light of the crucial observations reported above, we now propose a mechanism for the jet destabilization. First, the absence of wings for large $l$ shows that the symmetry 

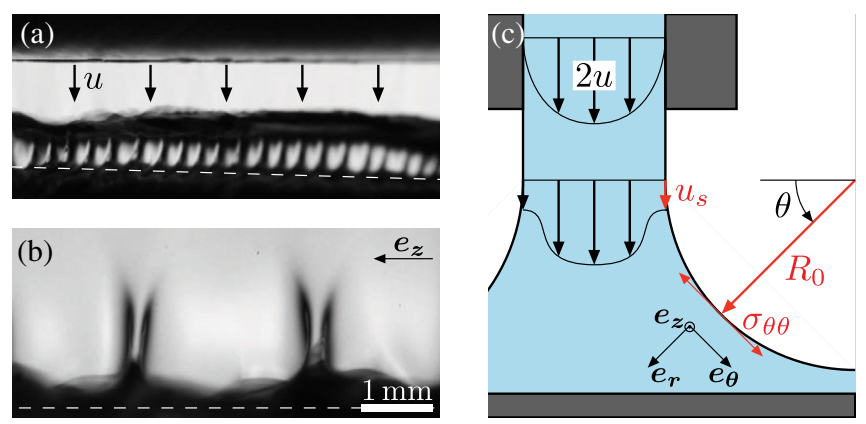

FIG. 3 (color online). (a),(b) Angle and perpendicular view of the transverse destabilization of a liquid sheet (elongated jet) impacting on a plate. The dashed line indicates the plate surface. (c) Schematics of the destabilization mechanism. The liquid is sheared at the nozzle wall and develops an elastic tension $\sigma_{\theta \theta}$ along the streamlines. On impact, where the air-liquid interface curves $\left(R_{0}>0\right)$ due to the deflection by the plate, $\sigma_{\theta \theta}$ promotes the destabilization of the interface in the perpendicular direction $\boldsymbol{e}_{z}$. By contrast, the velocity $u_{s}$ at the jet surface (set by the viscous relaxation of the shear, as the liquid travels from the nozzle to the plate) stabilizes the interface due to an inertial effect.

breaking is not an intrinsic destabilization of the jet (i.e., independent from impact). Second, the azimuthal destabilization is transverse to the axial folding (viscous buckling $[23,24]$ ) observed for viscous jet impacts [25], and, as already mentioned, we could not observe symmetry breaking with viscous Newtonian liquids. This suggests that the symmetry breaking is intrinsically viscoelastic, as the purely elastic instability reported for Couette flows. The general destabilizing mechanism is the same as that identified by [16-18]: the presence of a tension (hoop stress) tangent to curved streamlines. In the present impact configuration this tension develops when the liquid is sheared in the nozzle, as suggested by the plate-to-nozzle distance dependence of the destabilization. Close to the plate, where the liquid streamlines are deflected, the interface is curved with a radius $R_{0}$ [see Fig. 3(c)]. Since the destabilization is also observed for an impacting liquid sheet [see Figs. 3(a) and 3(b)], we consider a simplified base state geometry that is invariant along the transverse direction $\boldsymbol{e}_{z}$. The (unstable) equilibrium along the radial direction $\boldsymbol{e}_{\boldsymbol{r}}$ yields $-\rho u_{s}^{2} / R_{0}=\operatorname{div} \overline{\overline{\mathbf{\sigma}}} \cdot \boldsymbol{e}_{\boldsymbol{r}}$, where $u_{s}$ stands for the liquid velocity at the interface [in $r=R_{0}$; see Fig. 3(c)]. There is thus a radial gradient of the normal stress $\partial_{r} \sigma_{r r}=\left(\Delta \sigma-\rho u_{s}^{2}\right) / R_{0}$ that is related to the normal stresses difference $\Delta \sigma=\sigma_{\theta \theta}-\sigma_{r r}$. The stability of a liquid interface directly depends on the sign of this tension ("negative pressure") gradient, whether the latter results from inertial, gravitational (e.g., Rayleigh-Taylor [30]) or viscous effects (e.g., Saffman-Taylor [31]). For a negative tension gradient $\left(\partial_{r} \sigma_{r r}<0\right)$ the interface is stable. Conversely, for a positive gradient $\left(\partial_{r} \sigma_{r r}>0\right)$ there is a positive feedback between the interface deformation and the liquid flow beneath that amplifies the initial deformation.
Following [32] we make an analogy with a RayleighTaylor instability with an effective gravity $\partial_{r} \sigma_{r r} / \rho$ (see also [33]). We consider a perturbation of the interface position in the form $R=R_{0} \mathrm{e}^{\mathrm{i} k z+\omega R_{0} \theta / u_{s}}$, i.e., a perturbation that is advected by the steady velocity $u_{s} \boldsymbol{e}_{\boldsymbol{\theta}}$, and obtain

$$
\rho \omega^{2}=\frac{\Delta \sigma-\rho u_{s}^{2}}{R_{0}} k-\gamma k^{3} .
$$

As already noted, the interface is unstable ( $\omega$ real) only if $\Delta \sigma$ is positive, but also larger than the stabilizing centripetal acceleration term $\rho u_{s}^{2}$. In that case, the most unstable wave number and growth rate are $k_{m}^{2}=\left(\Delta \sigma-\rho u_{s}^{2}\right) / 3 \gamma R_{0}$ and $\omega_{m}^{2} \simeq 0.38 \sqrt{\left(\Delta \sigma-\rho u_{s}^{2}\right)^{3} / \gamma \rho^{2} R_{0}^{3}}$, respectively. To apply this formalism to our situation, we first relate the normal stress to the shear at the nozzle inner wall, i.e., $\Delta \sigma \sim \eta \lambda{\dot{\gamma_{0}}}^{2}$ (assuming in agreement with [34] that the solution behaves as an Olroyd-B liquid), where the shear rate at the nozzle wall expresses as $\dot{\gamma_{0}}=4 u / a$ (assuming a Poiseuille flow with uniform viscosity). Second, the velocity $u_{s}$ at the surface of the jet differs from the mean flow velocity $u$ and depends on the distance $l$ to the nozzle, as schematized in Fig. 3(c). Indeed, immediately at the nozzle exit $u_{s}=0$ by continuity. At large distance, the velocity profile in the jet cross section relaxes to a plug flow, which, from Eq. (1), stabilizes the interface (the elastic stresses relaxation is subdominant here since $l / u \lambda \ll 1)$. Since we observed no thickening of the jet, we assume that the relaxation of the jet velocity is mainly due to viscous effects. Following [35] the surface velocity is $u_{s} \simeq$ $3\left(\eta l / \rho u a^{2}\right)^{1 / 3} u$ (the velocity in the jet equalizes over a typical penetration depth $\sqrt{\eta l / \rho u_{s}}$ at a value which is set by the initial velocity gradient at the wall $4 u / a)$. Gathering all these expressions, and using $R_{0} \sim a$ (valid for the base state of both cylindrical jets and liquid sheets $[4,10]$ of which we consider here the linear destabilization), yields

$$
n \simeq k_{m} a=N \frac{u}{U} \sqrt{1-\left(\frac{u}{U}\right)^{-2 / 3}}
$$

(valid for $u>U$ ), where

$$
N=\frac{3^{5 / 2}}{16} \sqrt{\frac{\rho a l^{2}}{\gamma \lambda^{2}}}, \quad U=\frac{27}{64} \sqrt{\frac{\rho a^{2} l^{2}}{\eta \lambda^{3}}} .
$$

Equation (2) is in agreement with all the experimental trends discussed above. It predicts a threshold velocity for symmetry breaking $U \propto l / \lambda^{3 / 2}$ that increases with $l$ and decreases with $\lambda$. Equation (2) also expresses that for $u \gg U$, the number of wings $n \sim N u / U$ is proportional to $u$. It is compared to all our measurements, with both straight and convergent nozzles, in Fig. 4 [see also Fig. 2(a) for a direct comparison]. The measurements are scattered around Eq. (2), which we attribute to the simplifications of 


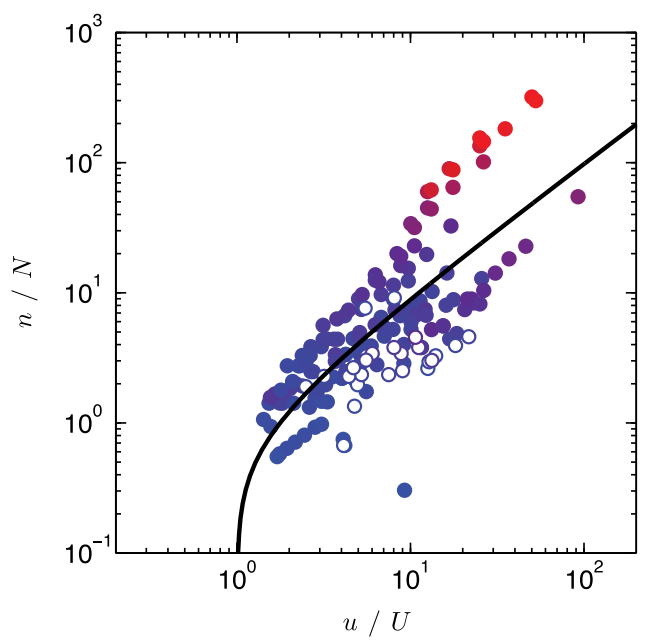

FIG. 4 (color online). Dimensionless number of wings $n / N$ versus dimensionless jet velocity $u / U$, where $N$ and $U$ are computed from the parameter-free expressions of Eq. (3). All the experiments (with $a=0.77-1.38 \mathrm{~mm}, u=1.33-16.3 \mathrm{~m} / \mathrm{s}$, $l=0.5-15.0 \mathrm{~mm}$ and $\lambda=7.3-17.6 \mathrm{~ms}$ ) are presented. The solid line represents Eq. (2). The filled (hollow) circles stand for straight (convergent) nozzles. The color code represents the absolute number $n$ (from 0 , dark blue, to the maximal value 34, dark red).

the model regarding the base state and the nonlinearities arising from high amplitude saturated states. However, in spite of these simplifications, Eq. (2) collapses most of the data and captures both the order of magnitude and the trends for $n$ (with $u, l$, and $\lambda$ ) with no adjustable parameter, which further supports the viscoelastic mechanism we invoke.

We conclude by highlighting the large-scale consequences of the jet destabilization. Figure 5 shows suspended (Savart) and spreading liquid sheets, formed by a viscoelastic jet impacting on a small disk (with radius $2 a$ ), and on a large plate, respectively. It shows that the symmetry breaking extends to the whole of the sheets: the shapes of both the Savart sheet and the hydraulic jump on the plate are prescribed by the jet instability. In both situations the local radius (distance from the impact point to the edge or jump) is smaller in the directions of the wings [visible in Figs. 5(d) and 5(f), and hidden but also true in 5(a), 5(b), and 5(c)]. This means that both the sheet momentum and flow rate, which respectively set the sheet radius [36] and the hydraulic jump radius in the viscous limit [37], are reduced in the directions of the wings relatively to other directions. Interestingly, for Savart sheets the jet instability also sets the number of ejection sites initiating the liquid fragmentation. However, contrarily to previous studies, the polygonal shapes are not forced by an anisotropy in the impact geometry [1,36,38], nor do they result from the edge or jump proper dynamics [39-41], but rather arise from the spontaneous symmetry breaking on impact. We finally note that the destabilization of the jet is probably not always steady. One expects that unsteadiness

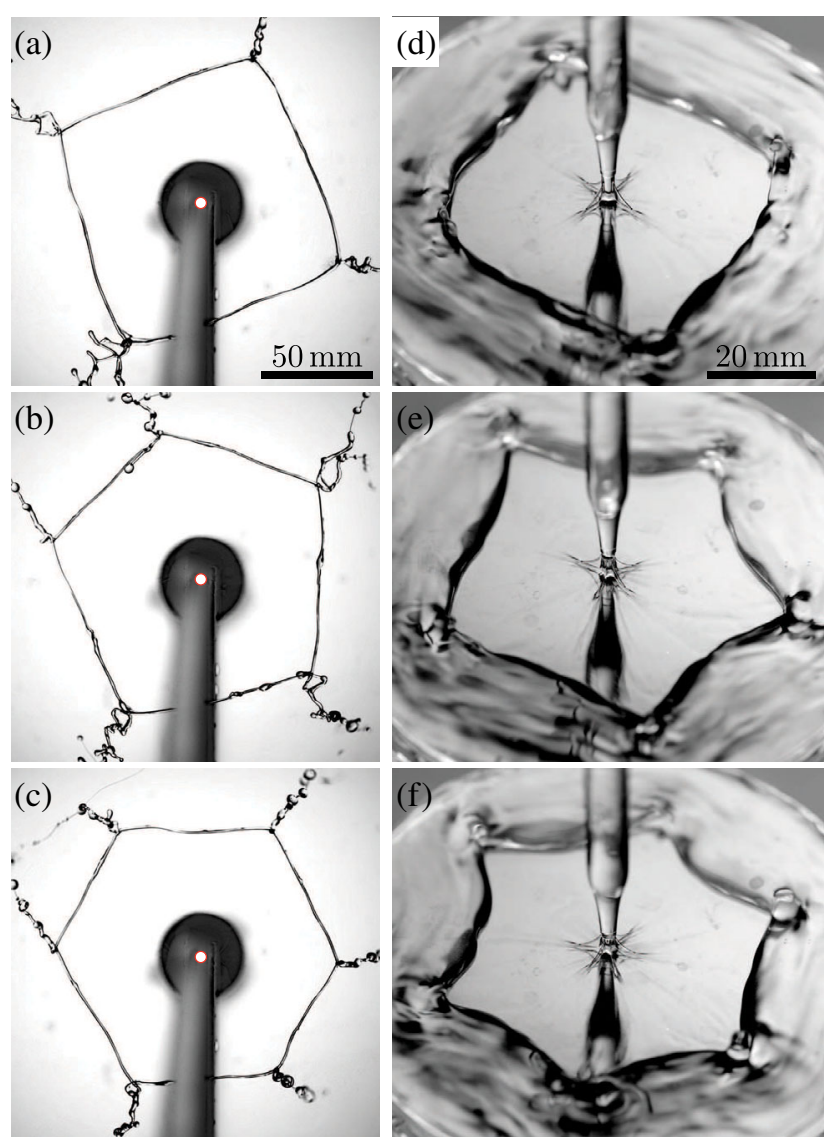

FIG. 5 (color online). Large-scale consequences of the jet symmetry-breaking for $n=4$ to 6. (a),(b),(c) Suspended (Savart) liquid sheets formed by the impact on a disk twice as large as the jet (represented by the white disk in the center). The sheet radius (hence the sheet momentum) is minimal in the wings directions, and the liquid is ejected at the vertices of the sheet. (d),(e), (f) Hydraulic jumps formed by the impact on a large plate. The radius of the hydraulic jump (hence the sheet flow rate) is also minimal in the wings directions.

and turbulence of elastic origin emerge at higher velocities or longer relaxation times. More generally, one might also wonder if other systems that build up normal stresses, like granular [42] or suspensions, could develop the same destabilization.

We thank L. Lebon and A. Lindner for useful discussions on polymer solutions, G. McKinley for useful discussions about the destabilization mechanism, and A. Duchesne for useful discussions on the hydraulic jump. This work was sponsored by the Agence Nationale de la Recherche (Freeflow ANR-11-B504-001-01).

*henri.lhuissier@univ-paris-diderot.fr

[1] F. Savart, Ann. Chim. 54, 55 (1833).

[2] F. Homann, Z. Angew. Math. Mech. 16, 153 (1936).

[3] G. I. Taylor, Proc. R. Soc. A 259, 1 (1960).

[4] G. I. Taylor, Phil. Trans. R. Soc. A 260, 96 (1966). 
[5] E. Watson, J. Fluid Mech. 20, 481 (1964).

[6] H. Lhuissier and E. Villermaux, J. Fluid Mech. 693, 508 (2012).

[7] G. I. Taylor and L. Howarth, Proc. R. Soc. A 253, 289 (1959).

[8] C. Clanet and E. Villermaux, J. Fluid Mech. 462, 307 (2002).

[9] S. Leach, G. Walker, A. Smith, I. Farmer, and G. I. Taylor, Phil. Trans. R. Soc. A 260, 295 (1966).

[10] X. Liu, L. Gabour, and J. Lienhard, J. Heat Transfer 115, 99 (1993).

[11] H. Lhuissier and E. Villermaux, J. Fluid Mech. 714, 361 (2013).

[12] E. Villermaux, V. Pistre, and H. Lhuissier, J. Fluid Mech. 730, 607 (2013).

[13] M. Negri, H. Ciezki, and S. Schlechtriem, Prog. Energy Combust. Sci. 4, 271 (2013).

[14] L.-J. Yang, Q.-F. Fu, Y.-Y. Qu, B. Gu, and M.-Z. Zhang, Int. J. Multiphase Flow 39, 37 (2012).

[15] R. Larson, Rheol. Acta 31, 213 (1992).

[16] E. Shaqfeh, Annu. Rev. Fluid Mech. 28, 129 (1996).

[17] P. Pakdel and G. H. McKinley, Phys. Rev. Lett. 77, 2459 (1996).

[18] M. A. Fardin, D. Lopez, J. Croso, G. Grégoire, O. Cardoso, G. H. McKinley, and S. Lerouge, Phys. Rev. Lett. 104, 178303 (2010).

[19] The shear viscosity is measured in a cone/plane rheometer (Anton Paar MCR 500). The solution is found to be slightly shear thinning with a viscosity decreasing from 6.5 to $3.5 \mathrm{mPa} \mathrm{s}$ in the range of shear rate $10^{2}-7 \times 10^{3} \mathrm{~s}^{-1}$.

[20] When it drips, the drop entrains a long cylindrical liquid filament with a uniform radius that exponentially decays with time. $\lambda$ is measured by fitting the law $\mathrm{e}^{-t / 3 \lambda}$ to the radius time evolution [21].

[21] V. Tirtaatmadja, G. H. McKinley, and J. Cooper-White, Phys. Fluids 18, 043101 (2006).

[22] See Supplemental Material at http://link.aps.org/ supplemental/10.1103/PhysRevLett.113.194502 for [brief description].

[23] G. Barnes and R. Woodcock, Am. J. Phys. 26, 205 (1958).
[24] N. Ribe, M. Habibi, and D. Bonn, Annu. Rev. Fluid Mech. 44, 249 (2012).

[25] Note that viscous buckling can lead to azimuthal destabilizations when the flow has azimuthal or radial centripetal components [26] which are enhanced by viscoelasticity [27-29], but this is not the case here.

[26] T. Benjamin and T. Mullin, J. Fluid Mech. 195, 523 (1988).

[27] G. Beavers and D. Joseph, J. Fluid Mech. 81, 265 (1977).

[28] A. Kumar and M. Graham, J. Non-Newtonian Fluid Mech. 89, 1337 (2000).

[29] T. Podgorski and A. Belmonte, J. Fluid Mech. 460, 337 (2002).

[30] J. Lord Rayleigh, Proc. R. Soc. A 14, 170 (1883).

[31] P. Saffman and G. I. Taylor, Proc. R. Soc. A 245, 312 (1958).

[32] M. Graham, Phys. Fluids 15, 1702 (2003).

[33] L. Aitken and S. Wilson, J. Non-Newtonian Fluid Mech. 49, 13 (1993).

[34] A. Zell, S. Gier, S. Rafaï, and C. Wagner, J. Non-Newtonian Fluid Mech. 165, 1265 (2010).

[35] A. Sevilla, J. Fluid Mech. 684, 204 (2011).

[36] G. I. Taylor, Proc. R. Soc. A 253, 296 (1959).

[37] T. Bohr, P. Dimon, and V. Putkaradze, J. Fluid Mech. 254, 635 (1993).

[38] E. Dressaire, L. Courbin, J. Crest, and H. A. Stone, Phys. Rev. Lett. 102, 194503 (2009).

[39] C. Ellegaard, A. Hansen, A. Haaning, K. Hansen, A. Marcussen, T. Bohr, J. Hannsen, and S. Watanabe, Nature (London) 392, 767 (1998).

[40] R. Buckingham and J. Bush, Phys. Fluids 13, S10 (2001).

[41] J. M. Gordillo, H. Lhuissier, and E. Villermaux, J. Fluid Mech. 754, R1 (2014).

[42] X. Cheng, G. Varas, D. Citron, H. M. Jaeger, and S. R. Nagel, Phys. Rev. Lett. 99, 188001 (2007). 


\section{Supplemental material}

\section{A. Wing confinement at short distance}
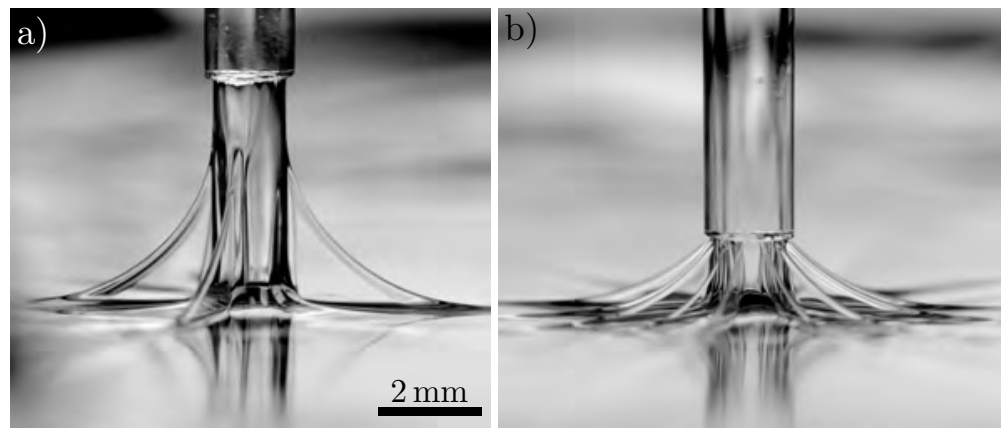

Figure 1: Perpendicular impact onto a glass plate. a) For large plate-to-nozzle distance $l$ the wingnes height is smaller than $l$. b) For short $l$, the wings are confined and their size is prescribed by $l$.

\section{B. Facing jets and oblique impacts}

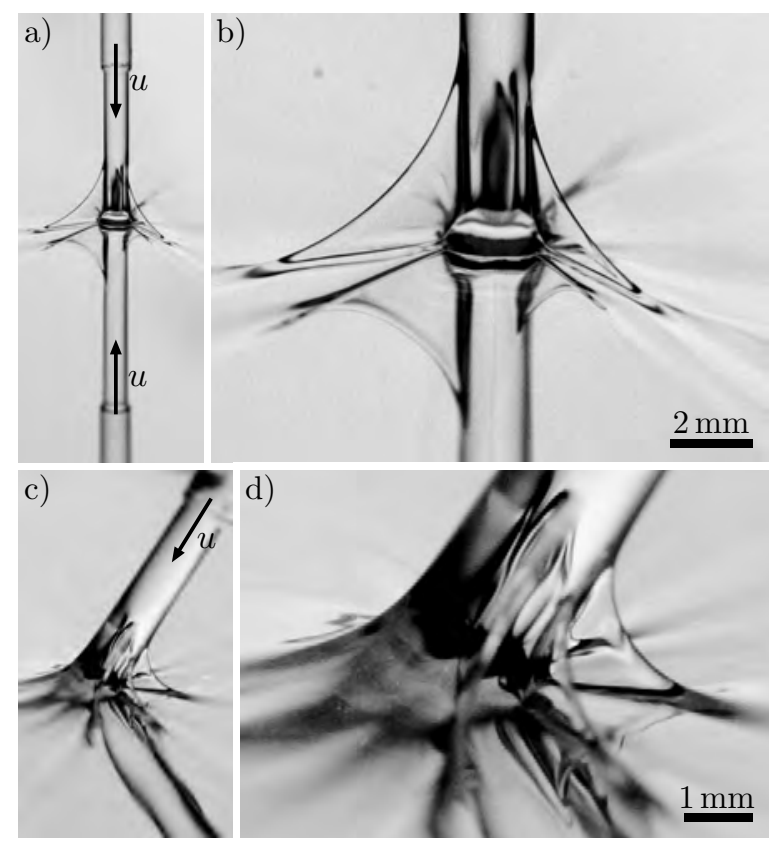

Figure 2: a-b) Impact of two facing jets (i.e. facing coaxial jets with same size and opposite velocities). The jets destabilize at the impact, as for a jet impacting onto a solid plate. c-d) Oblique impact of a jet onto a solid plate. The wings are larger on the (righthand) side where the angle of deflection and the radius of curvature of the streamlines are the largest and the smallest, respectively (the jet image at the bottom is a reflection on the sheet). 